\title{
La nécessaire politique en faveur du développement des capacités d'innovation dans le secteur agroalimentaire au Mali
}

\author{
The Need for a Policy Focused on the Development of Innovation \\ Capacities in the Malian Agri-Food Sector
}

\author{
Ismaïla El Moctar Haïdara ${ }^{1}$
}

${ }^{1}$ Laboratoire de Recherche sur l'Industrie et l'Innovation, Université du Littoral Côte d'Opale, ismahaid@yahoo.fr

RÉSUMÉ. La problématique de l'alimentation et de la nutrition demeure un des défis importants que les pays en développement (PED), notamment le Mali, doivent relever. De nombreuses tentatives d'innovations technologiques ont été réalisées dans le cadre de la production et la transformation des produits agricoles pour répondre aux besoins alimentaires des populations. Mais les objectifs ne sont pas atteints. La question à laquelle nous allons répondre dans cet article est de savoir dans quelle mesure le Mali peut mettre en place des politiques en faveur du développement des capacités d'innovation dans le secteur agroalimentaire. Cette question se justifie par le nombre d'acteurs (insuffisants) ainsi que la nature des interactions (faibles) susceptibles à favoriser la construction et/ou le renforcement des capacités et des processus d'innovations technologiques (et non technologiques) dans ce secteur.

ABSTRACT. The issue of food and nutrition remains one of the major challenges facing developing countries (DCs), particularly Mali. Many attempts at technological innovation have been made in the production and processing of agricultural products to meet the food needs of populations. But the objectives have not been achieved. The question we will answer in this article is to what extent Mali can put in place policies to promote the development of innovation capacities in the agri-food sector. This question is justified by the number of (insufficient) actors and the nature of (weak) interactions likely to foster the construction and/or strengthening of technological (and non-technological) innovation capacities and processes in this sector.

MOTS-CLÉS. Agroalimentaire, Capacités d'innovation, Développement, Politique publique, Mali.

KEYWORDS. Agri-food, Innovation capacity, Development, Public policy, Mali.

\section{Introduction}

La problématique de l'alimentation et de la nutrition demeure parmi les défis importants que les pays en développement (PED), notamment le Mali, doivent relever. De nombreuses tentatives d'innovations technologiques ont été réalisées dans le cadre de la production et la transformation des produits agricoles pour répondre aux besoins alimentaires des populations. Mais les objectifs n'ont pas été atteints eu égard aux résultats de l'enquête nationale sur la sécurité alimentaire et nutritionnelle (ENSAN), effectuée en septembre 2018, montrant que 19,1\% des ménages maliens sont affectés par l'insécurité alimentaire, contre $23,3 \%$ en 2017, soit une baisse de $21 \%$. Cette baisse est à relativiser: la situation alimentaire est fortement dépendante des aléas climatiques, de la fluctuation des prix des produits alimentaires et de l'évolution de la conjoncture économique sousrégionale et internationale. Ces difficultés anéantissent souvent les efforts consentis et les résultats enregistrés. 46,7\% de la population des régions du nord du pays (Gao, Tombouctou, Kidal, Taoudéni et Menaka) ${ }^{1}$ sont affectés par l'insécurité alimentaire. Par ailleurs, l'agroalimentaire est fortement tributaire des techniques culturales rudimentaires. $\grave{A}$ titre d'illustration, $72 \%$ des productions sont réalisées avec les charrues à traction animale, $17 \%$ avec les dabas et seulement $1 \%$ sont motorisées ${ }^{2}$. Cet état des lieux prouve les faibles capacités d'innovation des acteurs du secteur,

\footnotetext{
${ }^{1}$ Rapport de synthèse : Enquête Nationale sur la sécurité alimentaire et nutritionnelle au Mali, septembre 2018

2 Plan National d'investissement prioritaire dans le secteur agricole 2011-2015, Mali 
définies comme l'ensemble de processus d'apprentissage fondés sur l'accès au savoir et aux opportunités d'apprentissage et sur l'utilisation des savoirs (par les politiques d'emploi et d'innovation et par la stratégie des entreprises) [CAS 11] ; la richesse de ces capacités permettant l'émergence et la diffusion de l'innovation et la transformation des techniques et des modèles organisationnels.

La question à laquelle nous allons répondre dans cet article est de savoir dans quelle mesure le Mali peut mettre en place des politiques en faveur du développement des capacités d'innovation dans le secteur agroalimentaire. Cette question se justifie par le nombre d'acteurs (insuffisants) ainsi que la nature des interactions (faibles) contribuant à favoriser la construction et/ou le renforcement des capacités et des processus d'innovations technologiques et non technologiques dans ce secteur. Pour cela deux hypothèses sont retenues. 1/ Étant donné que la demande est croissante en produits agroalimentaires, le développement des capacités d'innovation peut augmenter la performance de ce secteur à court et moyen terme et répondre aux besoins alimentaires de la population du Mali. 2/ Étant donné que l'offre de produits agroalimentaires est insuffisante, le développement des capacités d'innovation dans l'agroalimentaire est nécessaire, d'où l'importance des politiques publiques en sa faveur pour augmenter qualitativement et quantitativement les produits agroalimentaires et satisfaire la demande locale.

Pour cela nous allons procéder en deux parties. L'accent est mis sur les capacités d'innovation dans le secteur agroalimentaire au Mali, en faisant ressortir les raisons des échecs des différentes politiques ou initiatives prises dans ce domaine. Nous présenterons dans un premier temps la littérature relative aux capacités d'innovation corrélées au développement économique. Ceci nous conduira à étudier le cas du Mali en établissant un état de lieux et les faiblesses des capacités d'innovation dans le secteur agroalimentaire. Puis, la seconde partie portera sur la nécessité de mettre en place des politiques en faveur du développement des capacités d'innovation dans le secteur agroalimentaire au Mali. Dans cette optique, nous évoquerons l'inadaptation des politiques publiques actuelles aux besoins de ce secteur. Ceci justifie notre analyse prospective des facteurs susceptibles de contribuer au développement des capacités d'innovation dans le secteur agroalimentaire au Mali.

\section{Capacités d'innovation dans le secteur agroalimentaire au Mali : constat d'échec}

\subsection{Capacités d'innovation et développement : une revue de la littérature}

Étant préoccupés par la satisfaction des besoins socioéconomiques de base, les pays en développement accordent une place prépondérante dans leurs politiques économiques à l'éducation, la santé, l'alimentation, l'accès à l'eau potable, l'énergie, l'assainissement, la sécurité, la résilience face aux problèmes climatiques, reléguant au second rang la politique spécialement dédiée à l'innovation. Ce qui fait que la construction des capacités d'innovation n'est pas considérée comme prioritaire. D'où la pertinence des analyses pour identifier leurs rôles, leurs places et leurs apports dans l'efficacité des politiques publiques. Pour cela, il est nécessaire de partir sur la base des travaux déjà réalisés dont ceux de Casadella et Uzunidis pour comprendre la genèse la construction des capacités d'innovation (schéma 1).Elles contribuent à créer des dynamiques interactives à travers la diffusion du savoir, les capacités d'apprentissage et d'adaptation de nouvelles technologies nécessaires à la performance des individus, des entreprises, des territoires et des économies nationales [CAS 18, P.4-5]. 


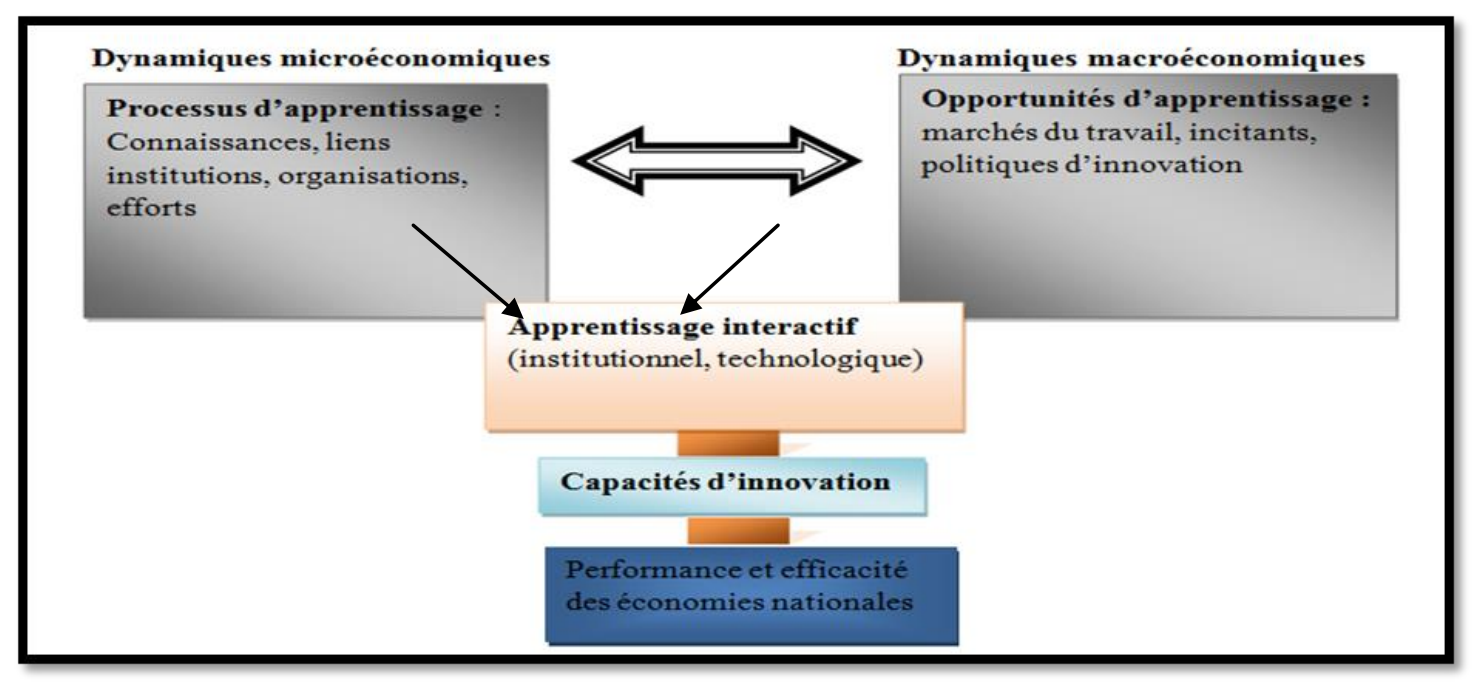

Figure 1. Genèse des capacités d'innovation (Casadella et Uzunidis, 2018)

La dynamique d'apprentissage interactif dans laquelle s'inscrivent toutes les parties prenantes combinant les connaissances existantes de base et l'intensité des efforts réalisés [COH 89] contribue à la construction des réelles capacités d'innovation, au renforcement de la performance et de l'efficacité des économies nationales. Alors le processus d'apprentissage va permettre par exemple à l'entreprise d'acquérir des connaissances (produits par les laboratoires de R\&D) et des savoir-faire spécifiques [LEB 93] [FOR 94] lui permettant d'optimiser son processus de production et être plus efficace.

La notion de «Système d'Innovation et de Construction de Compétences » a été élargie aux pays en développement, il y a une trentaine d'années [KIM 97] [HOB 97] [AMS 01] [BEL 97]. L’idée est d'utiliser le concept de «système d'innovation » pour comprendre le processus d'apprentissage et d'innovation et de s'en servir comme instrument pour comparer les performances technologiques et la promotion du développement économique de ces pays [CAS 15, P.49]. C'est dans ce cadre que Lundvall et al., et Kaplinski associent l'utilisation des connaissances à l'amélioration de la qualité de vie et de la réduction des inégalités qui demeurent des problèmes majeurs dans les PED [LUN 94] [KAP 09]. Les «capabilités », telles que définies par Amartya Sen, reliant les phénomènes de croissance aux processus d'expansion ou d'amélioration des capacités humaines à vivre dans des conditions socioéconomiques acceptables, sont indispensables [SEN 00]. Elles constituent à cet effet un préalable à la construction des compétences ([LUN 03, P.24] et à la croissance économique [CAS 14]. La prise en compte des aspects sociétaux ou environnementaux est alors impérative dans la mise en œuvre des processus d'innovation. Arocena et Sutz mettent l'accent sur les réalités locales «bricolées » dans les processus d'innovation, justifiant la négligence du potentiel scientifique et technologique ainsi que de leur trajectoire de développement [ARO 03]. C'est pour cette raison qu'ils considèrent l'approche «bottom-up » comme préalable à la construction de capacités d'innovation autour des dynamiques d'apprentissage localisées et couplées avec la mise en place de politiques éducatives et sociales. Ce qui nécessite des processus d'apprentissage et des opportunités au sein desquels les acteurs vont améliorer leurs capacités d'apprentissage par la recherche de solutions éventuelles [ARO 01]. Selon Lundvall, le rôle du système d'innovation et la construction des compétences est essentiel dans le développement industriel et serviciel ou dans l'adéquation entre le secteur formel et informel [LUN 13]. Cette dualité des économies en développement mérite une attention particulière, vu le rôle joué par le secteur informel dans la lutte pour la réduction des inégalités.

Il est impératif d'analyser les structures des pays en développement afin d'établir les liens entre l'innovation et le niveau de développement [NAY 02]. Ceci conditionne la pertinence des mesures prises par les autorités pour favoriser la construction des capacités d'innovation orientées vers 
l'obtention de résultats précis en matière de développement économique [JUM 01, P. 646]. En revanche, les inégalités (accès à la santé, à l'éducation, aux infrastructures de communication, etc.) constituent parfois un frein aux sources d'apprentissage [CAS 15, P.59]. Dans ce sens, l'innovation technologique peut contribuer à accentuer les inégalités de revenus et le chômage (p. ex. productions locales remplacées par les importations).

De plus, les interactions systémiques entre les différents acteurs sont souvent faibles voire inexistantes ou inappropriées [ARO 03], car les acteurs sont très souvent isolés, rendant difficile l'émergence des conditions de construction ou de développement des capacités d'apprentissage et d'innovation. L'isolement des unités de production peut s'expliquer par leur nombre insuffisant et le manque de cohérence infrastructurelle et stratégique, les privant ainsi des externalités (elles aussi faibles) produites par les laboratoires, les centres de recherche, les fournisseurs et clients, entravant ainsi les processus d'apprentissage technologique [CAS 17] [LAL 92]. À cela, s'ajoute l'asymétrie de l'information due aux faibles flux d'informations et de connaissances (lourdeur administrative, corruption, appropriation) qui circulent entre les acteurs ayant des difficultés à s'interconnecter ([MUC 03] [CHA 12]. Par ailleurs, les universités, les centres de formation d'ingénieurs et les laboratoires de R\&D sont en nombre insuffisant ; insuffisamment équipés, ils restent très éloignés des lieux de production [CHA 12]. A ceci s'ajoute l'inadéquation entre les formations universitaires et les besoins du marché qui crée des effets d'éviction des activités de recherche au profit de l'enseignement et de l'encadrement conduisant à une formation plus abstraite. Ogbu et al. justifient, en effet, l'insuffisance de capacités technologiques par les faibles capacités de production, la quasiabsence d'apprentissage technologique dans l'organisation de la production ainsi que par la faiblesse de liens inter-entreprises [OGB 95]. Cela se répercute sur l'incapacité à réaliser des innovations d'adaptation aux conditions sociotechniques locales (voir schéma 2).

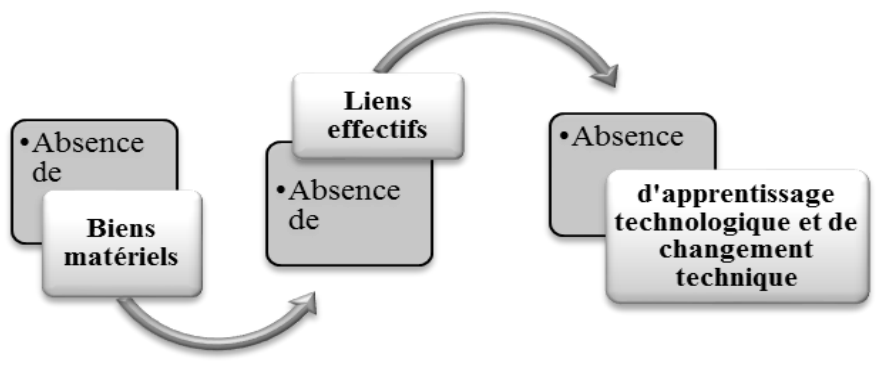

Figure 2. Faiblesse des bases productives et absence d'opportunités d'apprentissage (Auteur à partir d'Ogbu et al., 1995)

Selon Lall, dans certaines économies en développement, la fragile structure économique provient d'un ensemble inadapté d'incitants relatifs à l'environnement macroéconomique et aux politiques industrielles, d'institutions jalons de la structure technologique nationale et de capacités, liées aux ressources de l'activité technologique, des compétences techniques, de la finance et à l'accès à l'information locale et étrangère [LAL 92].

D'autres difficultés plus globales se greffent à la problématique du développement [CHA 12]. Il s'agit entre autres des problèmes :

- de capacités technologiques liées aux structures productives marquées par des faiblesses industrielles, des marchés et par une forte dépendance aux importations, anéantissant le développement de toute industrie locale ;

- d'informalité prédominante et d'inter-connectivité des réseaux. Le paradoxe étant que les relations sociales fortes coexistent avec leur faible mobilisation pour la production de richesses. Ce qui dénote d'une sous-estimation ou d'une ignorance de la force des relations sociales dans le cadre de l'activité économique. La mise à profit des relations sociales peut contribuer à baisser les coûts de 
transaction [WIL 81] et éliminer certaines barrières qui peuvent entraver la circulation des informations, des capitaux et de la main-d'œuvre ;

- institutionnels et leur faible fonctionnalité dans le domaine de l'accompagnement des processus d'innovation ou de gouvernance (corruption, clientélisme, absence ou incohérence de politique publique, non prise en compte de l'entrepreneuriat dans la construction des capacités d'innovation);

- de transition et de lock-in liés aux caractéristiques socio-historiques de dépendance consécutives à l'histoire coloniale, au développement des firmes globalisées, aux régimes politiques et religieux, aux réalités sociales et culturelles (constituant un frein à l'émergence de la construction de toute capacité d'innovation au niveau local, sous-régional et continental).

- Oyelaran-Oyeyinka pose cinq conditions pour la réalisation d'une dynamique d'apprentissage ayant pour objectif la constitution de capacités d'innovation en relation avec l'élévation du niveau du développement des forces de production :

- une variété de liens (formels et informels, forts et faibles) nécessitant offreurs, producteurs et fournisseurs de services ;

- une propension à la coopération et à la collaboration entre les différents acteurs ;

- un large nombre d'acteurs diffusant l'information et la connaissance nécessaire à la production de biens et services;

- une propension à l'incitation d'initiatives privées par la demande publique,

- un fort réseautage local-global formel et/ou informel [OYE 97].

Ces interactions sont capitales dans la mesure où différents types de connaissances vont pouvoir se combiner pour créer de nouvelles trajectoires et de nouvelles connaissances [CAS 17, P.16]. L'innovation englobe ainsi l'utilisation des connaissances et le renforcement des capacités technologiques. Le développement est considéré comme une fin mais aussi comme un moyen favorisant la construction de compétences nationales.

\subsection{Capacités d'innovation dans le secteur agroalimentaire au Mali}

\subsection{1. État des lieux des capacités d'innovation dans le secteur agroalimentaire au Mali}

Les écosystèmes sectoriels (dont l'agroalimentaire) permettent d'analyser les activités d'innovation et de production. L'agroalimentaire a toujours été au cœur des préoccupations des pouvoirs publics au Mali. C'est dans ce sens que les réflexions ont été menées pour mettre en place des cadres susceptibles de le doter de capacités lui permettant d'être le moteur de la croissance économique du Mali. Faire un état des lieux des capacités d'innovation dans ce secteur nécessite un rappel historique des politiques publiques en faveur de son développement. Cela permettra d'identifier ses capacités d'innovation et de comprendre son état actuel.

\subsubsection{Rappel historique des politiques publiques en faveur du développement agricole et alimentaire}

Pays à vocation agro-sylvo-pastoral, les différents gouvernements qui se sont succédés à la tête du Mali, des années 1960 à nos jours, ont élaboré différentes politiques en faveur du développement de tous les secteurs d'activité en général et particulièrement du secteur primaire. Sur ce plan, les politiques avaient pour objectif de satisfaire les besoins alimentaires des populations. Cela s'est concrétisé, durant la période 1960-1980, par la création de zones et opérations de développement économique dans les différentes régions du pays (Kayes, Bamako, Sikasso, Ségou, Mopti, Gao) et la promotion $\mathrm{du}$ «consommer malien » (riz, mil, poisson, coton, arachide, tomate, oignon, banane, 
etc.). Leur mode de gouvernance permet de comprendre la situation actuelle dans laquelle se trouve le secteur agroalimentaire.

Après son accession à l'indépendance, le Mali a opté pour le socialisme. Ce qui s'est traduit par la mise en place d'une économie planifiée de type dirigiste. Les grandes sociétés et entreprises d'État virent le jour et avaient le contrôle sur toute la sphère économique, notamment l'agriculture. Le contexte de l'après seconde guerre mondiale caractérisé par la dominance de la grande entreprise et le rôle important de l'interventionnisme étatique ont prévalu au choix de ce mode de gouvernance. Aucune place n'était laissée à l'initiative privée, à la concurrence et à l'innovation.

Les programmes de développement n'ont pas atteint leurs objectifs pour plusieurs raisons : mauvaise conception, insuffisance de compétences qualifiées, incohérence avec les attentes des populations et quasi dépendance du financement extérieur, le plus souvent insuffisant. La question de la gouvernance et de la performance des entreprises et des programmes de développement, corrélée à la paupérisation de toutes les classes sociales, les crises répétitives (chocs pétroliers, sécheresses, catastrophes climatiques), le fort endettement de l'État et les déséquilibres macroéconomiques ont montré les limites de l'interventionnisme étatique.

En réponse à l'excès d'endettement et au creusement des déséquilibres macroéconomiques des années 1980, la Banque Mondiale et le Fonds Monétaire International interviennent avec les Programmes d'Ajustement Structurel (P.A.S) appliqués par des politiques économiques conjoncturelles d'inspiration monétariste; l'idée de "l'État minimum » s'est imposée comme concept de base [CAS 15, P.13]. Les P.A.S avaient pour objectifs d'établir la stabilité macroéconomique, favoriser la croissance économique et de réduire la pauvreté. Ce qui exige des réformes majeures passant par l'assainissement des finances publiques, la réduction des dépenses publiques et du déficit budgétaire, la privatisation, la liquidation des entreprises publiques en banqueroute, les licenciements massifs dans la fonction publique et dans les entreprises publiques, la libéralisation de l'économie et son intégration dans l'économie mondiale. Le secteur agricole est également concerné par cette politique. Le programme de restructuration a concerné 35 entreprises publiques déficitaires dans les domaines de l'énergie, des télécommunications, des tabacs et allumettes, du chemin de fer, etc. 14 ont été privatisées et 12 liquidées (Huilerie cotonnière du Mali, Société Malienne d'Import-Export, chacune comptant plus 2000 travailleurs licenciés, la Société Africaine de Parfumerie et de Conditionnement) [MAL 87]. Les pertes d'emplois dans les secteurs publics et semi-publics portaient sur 1612 emplois permanents et $5053^{3}$ départs volontaires enregistrées entre 1990 et 1993. Le chômage s'est aggravé avec le blocage de recrutement dans la fonction publique, pénalisant particulièrement les jeunes diplômés à la recherche d'un emploi. Malgré la libéralisation économique, le secteur agroalimentaire malien peine à émerger d'où la nécessité du développement des capacités d'innovation dans l'élaboration de la politique économique.

\subsubsection{2. État des capacités d'innovation dans le secteur agroalimentaire au Mali}

L'innovation est la mise sur le marché ou dans le monde des affaires plus généralement d'un produit (bien ou service) ou d'un procédé nouveau ou sensiblement amélioré, d'une nouvelle méthode de commercialisation ou d'une nouvelle méthode organisationnelle dans les pratiques de l'entreprise, l'organisation du lieu de travail ou les relations extérieures [OCD 05]. Pour la mesurer, l'OCDE identifie des indicateurs suivants: les dépenses de R\&D, le nombre d'universités, d'institutions de formation technique et professionnelle, de centres technologiques, les laboratoires d'essai et de R\&D, agences publiques de promotion des sciences et des technologies et des types de

\footnotetext{
${ }^{3}$ Source : Groupe Banque Africaine de Développement, (1998), Programmes d'Ajustement Structurel, Rapport d'évaluation de la performance de projet (REPP), Mali, p. 37.
} 
liens entre les différents acteurs. La prise en compte de ces indicateurs permet de déterminer les capacités des différents acteurs à innover [OCD 05]. En ce qui concerne les PED, l'OCDE ajoute d'autres facteurs (socioéconomiques, politiques) afin de pouvoir les comparer aux pays développés. Ces pays n'ont pas les mêmes structures que les pays industriels et ils ne forment pas un ensemble homogène. L'existence de fortes disparités complique leur comparaison. Néanmoins, il est impérieux de prendre en compte la qualité et la quantité des ressources humaines [LUN 94] [KAP 09] [SEN 00], les liens entre les différents acteurs et les technologies de l'information et des communications [ARO 03], leur incorporation et leur utilisation à l'échelle du secteur agroalimentaire. Les capacités d'innovation du secteur étant les éléments qui lui permettent de tirer parti des possibilités offertes par le marché et de l'environnement socioéconomique et politique en général. Elles sont constituées par le savoir accumulé et incorporé dans les ressources humaines et les processus habituels utilisés dans le secteur.

Par ailleurs, nous avons identifié un certain nombre d'éléments non exhaustifs qui contribuent éventuellement à décrire les capacités d'innovation actuelles dans le secteur agroalimentaire. Toutefois, compte tenu de la nature et de l'inter-sectorialité (programmes de développement nationaux et internationaux regroupant à la fois agriculture, élevage, pêche, forêts, environnement, autres activités annexes ou connexes), il est difficile de dissocier l'agroalimentaire des autres secteurs. Au Mali en effet, l'accent est mis sur l'agroalimentaire en plus de l'éducation et la santé [ARO 03] [JUM 01] pour le développement de son économie.

Au niveau continental, selon les recommandations du sommet de Maputo (Mozambique) en 2003, tous les États africains se sont engagés à octroyer au moins 10\% de leurs budgets nationaux au développement du secteur agricole. L'objectif est double: franchir le cap de 6\% de croissance agricole annuelle pour atteindre l'Objectif du Millénaire pour le Développement (OMD) ${ }^{4}$ en matière d'alimentation et assurer le financement du secteur par les États en premier lieu, les fonds extérieurs venant en appui.

Pour le financement de la recherche scientifique, tous les États africains doivent consacrer $1 \%$ de leur PIB. Le Mali est en passe d'atteindre cet objectif en accordant actuellement $0,7 \%{ }^{5}$ de son PIB à cet effet. Grâce à cela, en 2017, près de 140 millions d'euros ont été mobilisés par l'Etat et le pays a reçu un appui financier de 394 millions d'euros de la part de ses partenaires internationaux. Ces fonds servent d'une part à financer la recherche agricole et d'autre part à l'achat des intrants et autres dépenses agricoles.

$\mathrm{Au}$ niveau sous-régional, la Communauté Économique des États de l'Afrique de 1'Ouest (CEDEAO) a mis en place le Plan Détaillé de Développement de l'Agriculture en Afrique / New Partnership for Africa's Development (PDDAA/NEPAD). Elle a mis en œuvre en 2009 le projet de « Pacte Economic Community of West African States Agricultural Policy ECOWAP/PDDAA » qui fédère les processus (sous régionaux et nationaux) et établit un cadre de partenariat entre les différents gouvernements dont celui du Mali, l'Union africaine, la CEDEAO, la profession agricole, les collectivités territoriales, les associations, les organisations de la société civile, le secteur privé ainsi que les Partenaires Techniques et Financiers en créant les conditions d'une meilleure coordination des politiques en matière de développement rural.

Au niveau national, la volonté politique des autorités maliennes pour faire du secteur primaire le fer de lance de l'économie, existe. Elle se traduit depuis 2014, par l'initiative du président de la République du Mali, Ibrahim Boubacar Kéita, d'allouer 15\% du budget public au développement du secteur agricole. En 2006, la Loi d'Orientation Agricole (LOA) a été adoptée. Elle crée un cadre juridique permettant de développer l'agriculture, faciliter les conditions d'accès au foncier agricole,

\footnotetext{
${ }^{4}$ https://www.un.org/sustainabledevelopment/fr/objectifs-de-developpement-durable/

${ }^{5}$ https://www.scidev.net/afrique-sub-saharienne/r-d/actualites/mali-odd-investissements.html 
offrir un climat des affaires garantissant les investissements. Elle est suivie par la formulation du cadre stratégique pour la croissance et la réduction de la pauvreté (CSCRP) servant de cadre de référence des politiques et stratégies gouvernementales en matière de lutte contre la pauvreté à moyen terme. Le Mail a signé avec ses Partenaires Techniques et Financiers (PTF) la Stratégie Commune d'Assistance Pays (SCAP) en vue d'orienter les actions de développement agricole vers une approche sectorielle par des appuis budgétaires sectoriels conséquents. À court terme, l'objectif est la mise en place d'instruments de gestion efficaces des investissements publics dans le secteur agricole à travers l'élaboration de la Politique de Développement Agricole (PDA).

Actuellement, le Mali dispose de 80 institutions de recherche dans tous les domaines. La recherche agricole est réalisée dans de centres de recherche (laboratoires, instituts), d'écoles, de centres d'expérimentation de nouvelles techniques culturales et de formation des cadres pour la valorisation des résultats de la recherche. En 2018, le Mali comptait 2068 enseignants dans l'enseignement supérieur (Institut National de Statistique du Mali, 2018), 29 chercheurs par million d'habitants, 9 publications par million d'habitants ${ }^{6}$. 169 brevets ont été déposés en 2017 (OMPI, 2018). Les autorités maliennes ont lancé en 2017, le Fonds Compétitif pour la Recherche et de l'Innovation Technologique (FCRIT) ${ }^{7}$ qui permet l'amélioration du financement de la recherche, l'organisation de la communauté des chercheurs, le renforcement de leurs capacités sur le plan équipement et formation, la promotion des partenariats public-privé en matière de R\&D. Il est destiné à financer les activités de recherche répondant aux besoins prioritaires du développement du pays par la conception et la mise en œuvre de projets innovants (agriculture, élevage, santé). Ce qui nécessite des partenariats entre les universités, les grandes écoles, les institutions de recherche, le secteur privé et les inventeurs isolés. L'objectif est de permettre aux assistants et attachés de recherche de développer des projets afin de mener des travaux de recherche correspondant à leur formation et valoriser les résultats des recherches.

Par ailleurs, les activités de production sont réalisées par 700000 exploitants ou entreprises agricoles et par 100000 autres dans les domaines de l'élevage, de la pêche, des forêts, de l'aviculture et autres activités du secteur primaire. Ils se partagent les 5,2 millions d'hectares qui ont été mis en valeur, soit $11,9 \%$ des 43,7 millions d'hectares ${ }^{8}$. Ce qui augure d'immenses potentialités qui peuvent être valorisées en vue de la modernisation du secteur agroalimentaire. Elles offrent de réelles opportunités d'affaires pour la création ou l'implémentation de nouvelles entreprises pouvant contribuer à créer de nouveaux produits, procédés, emplois, marchés, formes d'organisation du travail et par conséquent pouvant réduire les inégalités sociales et la pauvreté (Lundvall et Johnson, 1994).

\subsubsection{Les faiblesses des capacités d'innovation corrélées à l'archaïsme du secteur agroalimentaire au Mali}

Depuis cinq décennies, d'importants efforts ont été réalisés pour développer le secteur agricole au Mali à travers l'élaboration des plans quinquennaux de développement (plan de transition socialiste 1961-1965, programme triennal de redressement économique et financier 1970-1972, plan-projets d'investissements 1974-1978, programme, plan comptable destiné aux investisseurs étrangers 19811985, plan de sauvetage appelé ajustement structurel 1987-1991, schéma directeur de développement rural 1992-2010, cadres stratégiques de développement et programmes

\footnotetext{
${ }^{6}$ https://www.7x7.press/les-7-pays-d-afrique-ou-la-science-decolle

${ }^{7}$ http://www.ipr-ifra.edu.ml/wordpress/documents-utiles-sur-le-fonds-competitif-de-la-recherche-et-de-linnovationtechnologique/

${ }^{8}$ Ces informations sont issues du Plan National d'Investissement dans le Secteur Agricole, élaboré par le ministère du développement du Mali en 2014.
} 
d'investissement 2002-2020) [LEC 90]. Malgré cela, les capacités d'innovation restent faibles pour de multiples raisons. Pour déceler les faiblesses, nous allons mettre en avant les problèmes identifiés dans la revue de la littérature. Il s'agit entre autres du financement de la $R \& D$, l'accès à la santé et à l'éducation, du niveau de vie des populations, le manque, de l'insuffisance ou de l'inefficacité des infrastructures, du manque de compétences et d'interactions entre les acteurs.

Tout d'abord, de l'indépendance jusqu'à nos jours les programmes de R\&D agricoles sont restés tributaires des financements extérieurs (entre $51 \%$ et $87 \%$ des investissements totaux sur la période 1961-1991) [LAL 92] [LEC 90] et du manque d'intégration des capacités d'innovation dans les modèles et les stratégies de développement [GRE 01]. De plus en 2017, le Mali a pu mobiliser 140 millions d'euros destinés au financement de la recherche agricole, à l'achat des intrants, et a bénéficié d'un appui financier de 394 millions d'euros des partenaires internationaux. En plus d'être insuffisants, ces fonds sont orientés vers des activités improductives (coûts bureaucratiques trop lourds absorbant une large partie des fonds). Ils ne sont pas destinés directement à la satisfaction des besoins sociaux de base [LUN 94] [ARO 03] [KAP 09] [CAS 14][SEN 00]. Jusqu'à présent, il y a une insuffisance et parfois une absence d'infrastructures de base (routes, centres hospitaliers, réseaux de communication et d'eau potable, écoles, etc.). À cela s'ajoute la question de l'opacité de la gouvernance [CHA 12] qui justifie l'inefficacité ou l'absence d'optimisation des process ou des structures mises en place.

Malgré tous les efforts consentis en termes de financement de la recherche et de l'innovation dans le domaine agricole, les résultats peinent à émerger. À titre d'exemple, seulement $1 \%$ de la production agricole est réalisé par des machines et équipements modernes ${ }^{9}$. Par ailleurs, les résultats de la recherche ne sont pas valorisés à cause du manque d'interactions [OCD 95] [MUC 03] entre les centres de recherche, les universités et les entreprises du monde agricole. Chaque acteur ignore ce que l'autre peut lui apporter en termes d'expertise, de conseils, de nouveaux outils ou concepts en vue d'améliorer son activité ou d'innover. La conception des programmes, l'incohérence, la discontinuité, la gouvernance inappropriée, ainsi que l'absence, l'insuffisance et l'inefficacité des infrastructures, le manque d'évaluation des programmes précédents peuvent expliquer ces maigres résultats.

D'autre part, la faible mobilisation de l'épargne nationale (due au faible niveau de revenu, à la propension élevée à consommer, au taux d'épargne faible à cause de sa faible rentabilité) associée aux taux d'intérêt très élevés freinent les emprunts ainsi que les investissements dans les programmes de recherche et les technologies dans le secteur agroalimentaire. Elle motive d'un côté le choix de la plupart des acteurs (ménages et entreprises informelles) de thésauriser et garder leurs capitaux en dehors du système classique (à la maison le plus souvent); de l'autre côté, les conditions difficiles d'accès au crédit [LAL 92] privent les institutions financières de fonds importants pouvant servir à l'investissement. Cela est confirmé par le faible taux de bancarisation $16,5 \%$ en 2017 [BCE 18].

De plus, les politiques agricoles accordent une importance particulière aux cultures d'exportation (le coton) au détriment des produits vivriers (mil, maïs, pommes de terre et fruits) pour lesquels le pays dispose d'immenses potentialités. Seul le riz bénéficie d'une attention particulière à cause des infrastructures érigées (barrages hydro-agricoles) durant la seconde guerre mondiale et qui permettent une maîtrise totale ou partielle de l'eau dans certaines zones de l'office du Niger au centre du pays.

Nous pouvons aussi souligner l'insuffisance de centres de recherche, d'ingénierie, d'investissements en R\&D et de cadres compétents. Par exemple, le pays dispose de seulement deux

\footnotetext{
${ }^{9}$ Programme National d'Investissement Prioritaire dans le Secteur Agricole (2011-2015)
} 
écoles de formation d'ingénieurs : l'Institut Polytechnique Rural de Formation et de Recherche Appliquée (I.P.R./I.F.R.A.) de Katibougou et 1'École Nationale des Ingénieurs (E.N.I.) de Bamako qui forment près de 500 étudiants nationaux et étrangers par an. Ce nombre est insuffisant eu égard aux besoins réels du pays.

Sur le plan social, le niveau de vie des populations reste faible. En effet, en termes de développement humain le Mali se classe au $182^{\mathrm{e}}$ rang mondial sur 189 pays ${ }^{10}$ et occupe le $46^{\mathrm{e}}$ rang sur 53 pays Africains, selon le rapport 2018 du Programme des Nations Unies pour le Développement. Il est parmi les pays à IDH faible, d'où le taux élevé de la pauvreté soit, selon la Banque Mondiale, environ 42,7\% de la population en $2017^{11}$. Le faible niveau de développement peut expliquer les faibles capacités des acteurs à innover [LUN 94] [SEN 00] [NAY 02][ARO 03] [CHA 12][CAS 15]. La situation reste précaire et très préoccupante en raison de l'insécurité qui sévit dans le pays depuis 2012 et l'amplification des problèmes climatiques. Le schéma 3 montre l'évolution de l'indice du développement humain du Mali sur la période 1990-2017.

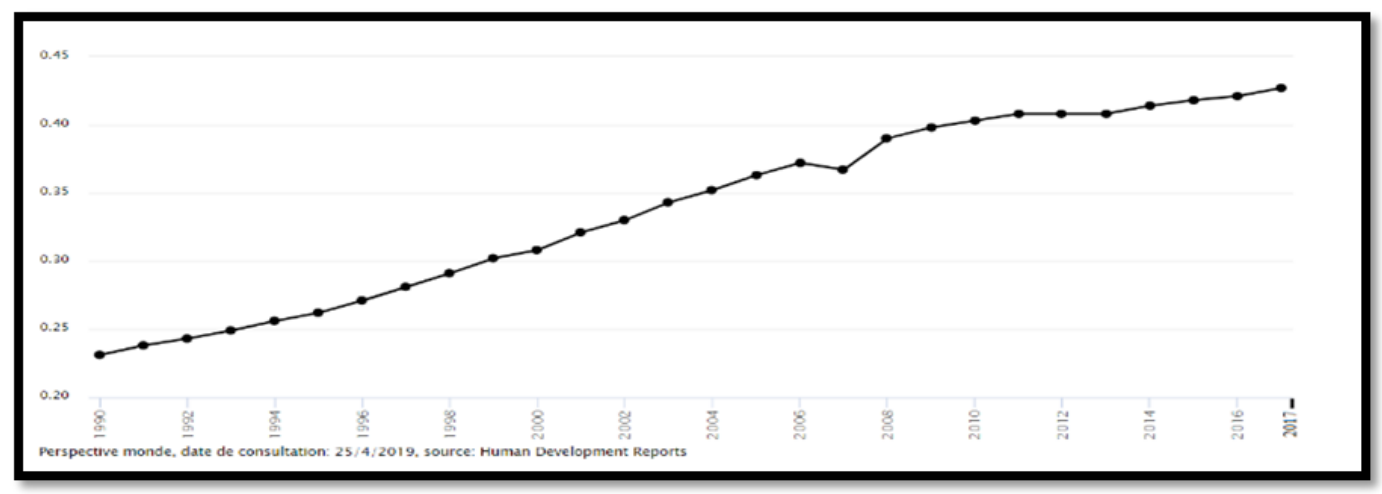

Figure 3. Évolution de l'IDH du Mali de 1990 à 2017

Nous constatons certes une amélioration de la qualité de vie des populations, mais le rythme d'évolution est lent et faible vu les efforts déployés et le fossé qui se creuse entre les régions du Sud et du Nord du pays et aussi à l'intérieur d'elles entre les couches sociales. Cet état des faits peut être expliqué entre autres par l'évolution des prix des denrées alimentaires durant l'année (schéma 4).

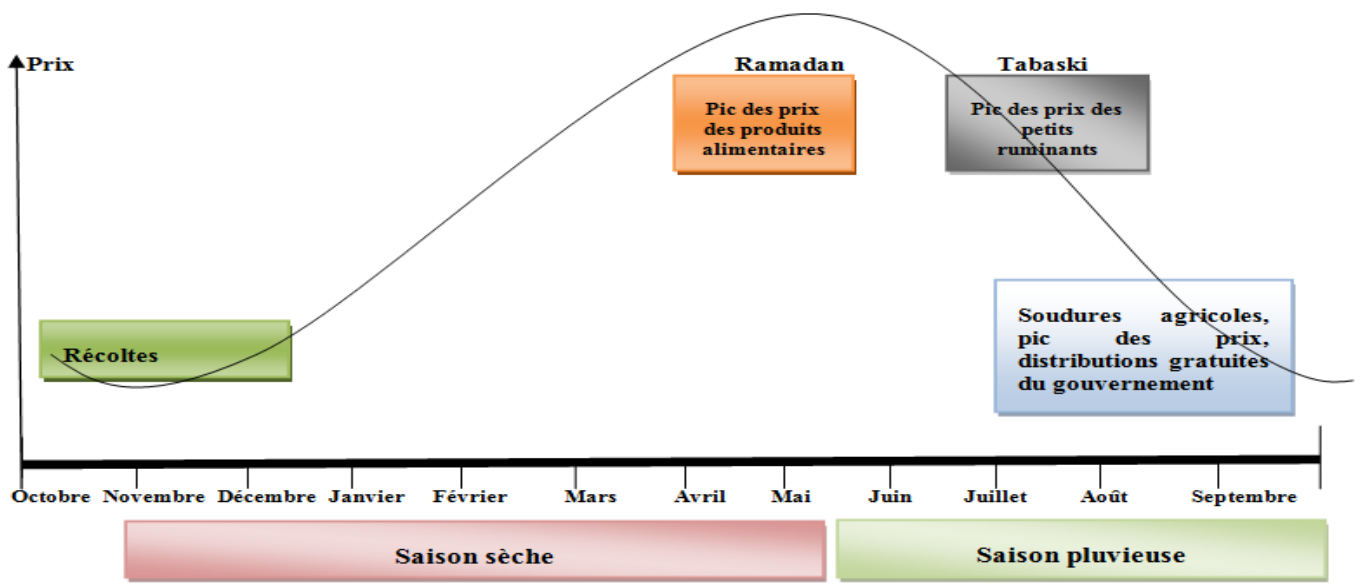

Figure 4. Évolution des prix des produits alimentaires et des petits ruminants durant l'année (Auteur à partir de fews.net/fr/west-africa/mali/food-security-outlook/)

\footnotetext{
${ }^{10}$ Les données sont issues de ce site https://www.agenceecofin.com/economie/1709-60037-classement-2018-des-paysafricains-par-indice-de-developpement-humain-pnud

${ }^{11}$ https://www.banquemondiale.org/fr/country/mali/overview
} 
Ce qui attire l'attention dans ce calendrier est que, chaque année, durant la « période de soudure » (juillet à octobre), le gouvernement du Mali procède à la distribution gratuite d'aliments aux populations vulnérables. Cela s'explique par le fait que, d'une part, les productions agricoles sont insuffisantes pour couvrir les besoins alimentaires des populations durant toute l'année d'où l'intervention de l'Etat et de ses partenaires; d'autre part, les productions agricoles (cultures de subsistances) sont très souvent bradées au moment des récoltes afin de faciliter l'accès à d'autres biens. Cette situation est exacerbée par la volatilité des prix des produits agricoles (forte baisse en période de récolte, forte hausse pendant les fêtes et la période de soudure), l'absence de routes, de moyens de conservations ou de transformation des produits et d'informations sur l'évolution du marché. Ce faisant, près du $1 / 5^{\mathrm{e}}$ de la population est concerné par l'insécurité alimentaire au Mali. La situation est très instable également en raison des aléas climatiques et des conflits récurrents autour de la gestion des ressources en eau, des pâturages et des terres agricoles notamment dans les régions du nord et du centre du pays depuis 2012. Le schéma 5 montre les relations difficiles qui ne peuvent s'instaurer entre besoins nutritionnels, sécurité alimentaire, production agricole et contexte sociopolitique instable.

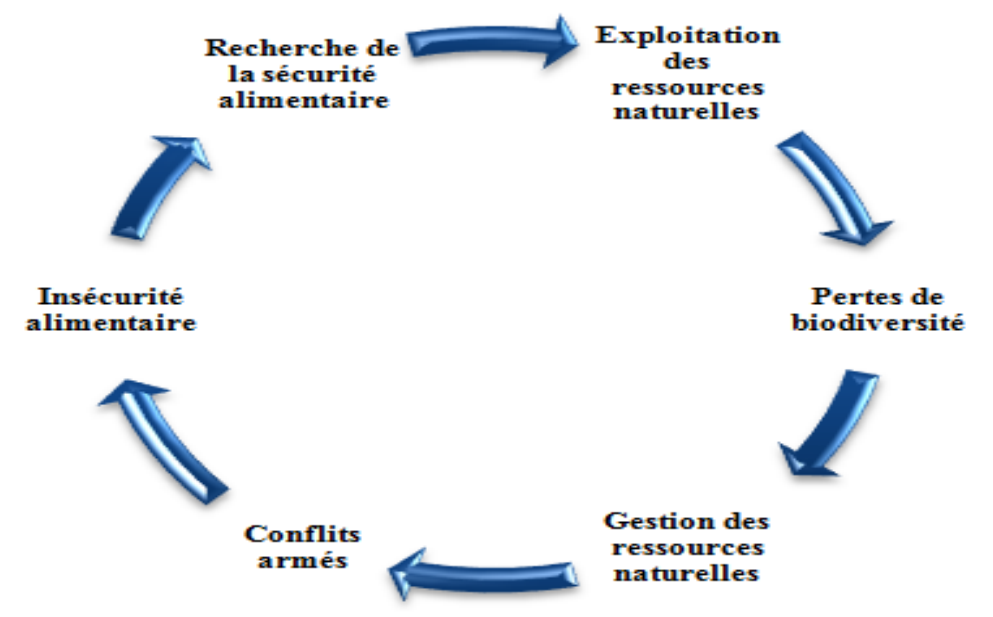

Figure 5. Le cercle vicieux de l'insécurité alimentaire au Mali

\section{La nécessaire politique en faveur du développement des capacités d'innovation dans le secteur agroalimentaire au Mali}

Dans cette partie de l'article, il s'agit de mettre en lumière les récentes politiques publiques en faveur du développement agricole au Mali. Cela est nécessaire afin de détecter les incohérences auxquelles se heurte le développement des capacités d'innovation dans le secteur agroalimentaire. Leur inadaptation peut se démontrer par l'absence ou la faible prise en compte de l'innovation comme clé de voûte de leur réussite. Ensuite, une analyse prospective des capacités d'innovation du secteur sera réalisée. Elle a pour but d'identifier les forces et les opportunités dont le Mali peut saisir ; les faiblesses et les menaces à prendre en compte dans le cadre des politiques publiques de renforcement des capacités d'innovation. Pour terminer, nous allons élargir la réflexion sur les conditions de réalisation de ces politiques.

\subsection{Politiques publiques inadaptées aux besoins du secteur agroalimentaire du Mali}

Nous allons partir des cadres et programmes élaborés durant la période 1992-2020 servant à la concrétisation des différentes politiques de développement des capacités agricoles. L'objectif est de faire ressortir leur inadaptation ou la non prise en compte de l'innovation en tant que facteur déterminant de leur réussite. Il s'agit :

- Du Schéma Directeur de Développement Rural (SDDR, 1992-2010) : c'est un instrument de la politique agricole permettant de développer des synergies entre les différents sous-secteurs du 
développement rural, de répondre aux attentes du monde rural et de contribuer au développement général du pays. C'est un cadre de dialogue flexible et évolutif de négociation entre l'Etat, les producteurs, la société civile et l'ensemble des partenaires au développement.

- Du Cadre Stratégique pour la Lutte contre la Pauvreté (CSLP, 2002-2006) : il a pour objectif de réduire la pauvreté de façon durable et d'améliorer les conditions de vie des couches les plus vulnérables de la population.

- De la Loi d'Orientation Agricole (LOA, 2006) : c'est un instrument directif et fédérateur de l'ensemble des dispositions législatives ou réglementaires touchant aux activités agricoles et périagricoles. Elle fixe les orientations de la politique de développement agricole et vise à sécuriser les terres agricoles, à développer les filières agricoles, à professionnaliser les acteurs, à diversifier les productions agricoles, à rendre compétitif le secteur agricole, à adopter un régime de protection sociale des agriculteurs adapté aux besoins et aux capacités du pays.

- Du Cadre Stratégique pour la Croissance et la Réduction de la Pauvreté (CSCRP, 2007-2011) : il a pour objectif d'impulser une croissance forte et soutenue (environ $7 \%$ par an), de réduire significativement la pauvreté par la relance des secteurs productifs, la décentralisation administrative, la promotion des initiatives du secteur privé et la bonne gouvernance économique et politique.

- Du Cadre Stratégique pour la Croissance, l'Emploi et la Réduction de la Pauvreté (CSCERP, 2012-2020) : il a pour objectif de consolider les acquis des cadres précédents tout en mettant l'accent sur la création d'emplois.

- De la Politique de Développement Agricole (PDA, 2011-2020): elle a pour objectifs de développer les investissements dans le secteur agricole, rendre compétitifs les produits sur les marchés domestiques et internationaux, développer la recherche agricole, renforcer les capacités des acteurs et assurer la sécurité alimentaire des populations.

- Du Programme National d'Investissement dans le Secteur Agricole (PNISA, 2011-2020) : c'est un cadre national de planification du développement du secteur agricole prenant en compte les besoins (fonctionnement, investissement et financement). Il intègre l'ensemble des projets et programmes du secteur. Il vise à faire du secteur agricole le moteur de l'économie nationale, assurer la sécurité alimentaire et nutritionnelle des populations urbaines et rurales, générer des emplois et des revenus significatifs dans une logique de développement durable.

\subsubsection{Schéma actuel des politiques publiques d'innovation dans le secteur agroalimentaire au Mali}

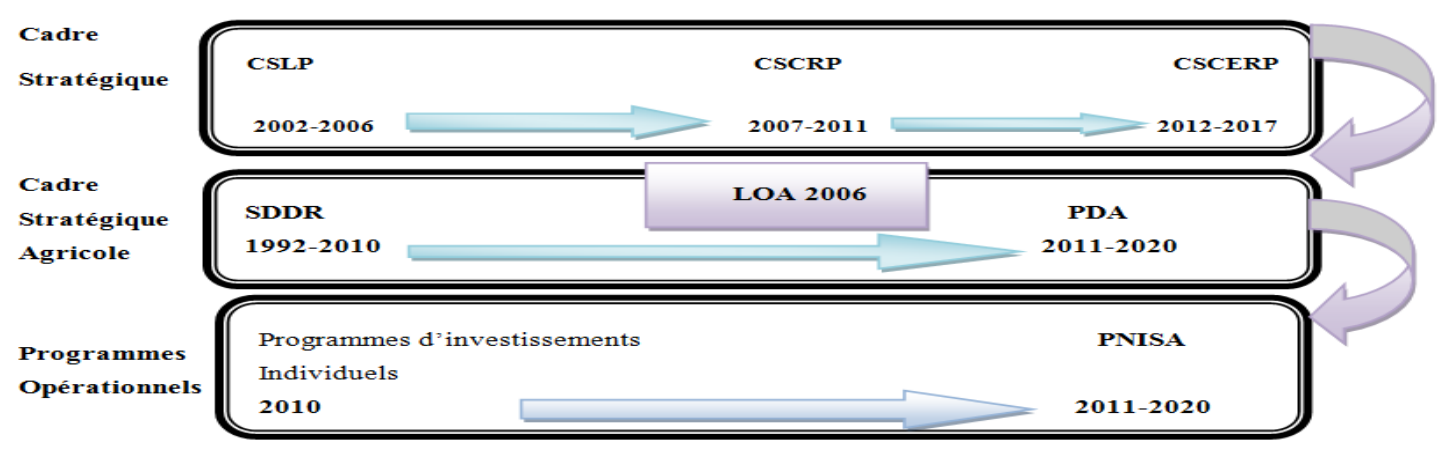

Figure 6. Cadres stratégiques et programmes opérationnels dans le secteur agricole au Mali (Rapport Pays du Suivi des Politiques Agricoles et Alimentaires en Afrique (SPAAA) Février 2013)

\subsubsection{Schéma des relations entre les différents acteurs}

À partir des politiques, nous remarquons un certain nombre d'éléments qui permettent d'établir un schéma de la nature des relations entre les acteurs de l'innovation dans ce secteur. Dans 
l'organisation des différentes activités, l'Etat est au cœur du système. Le développement du secteur agroalimentaire en général et des innovations en particulier se déroulent dans un cadre prédéfini et intégré dans des programmes nationaux, sous régionaux ou internationaux. Ces derniers peuvent fonctionner sous forme de "système » vu la nature des acteurs en présence et les organes ou entités qui les composent. Il y a des bases qui peuvent faciliter les interactions entre ces derniers. Mais le constat est qu'il y a des faibles interactions [OBG 95] [OYE 97] [CHA 12] entre les différents intervenants dans l'innovation au Mali. Le paradoxe est qu'ils entretiennent de relations fortes avec l'Etat, mais pas entre eux. Ce qui freine la diffusion des innovations susceptibles d'être produites par ces activités. Ce qui soulève la question de la gouvernance et la confiance (reconnaissance des intérêts communs) entre eux. De façon explicite, le schéma se présente ainsi (schéma 7) :

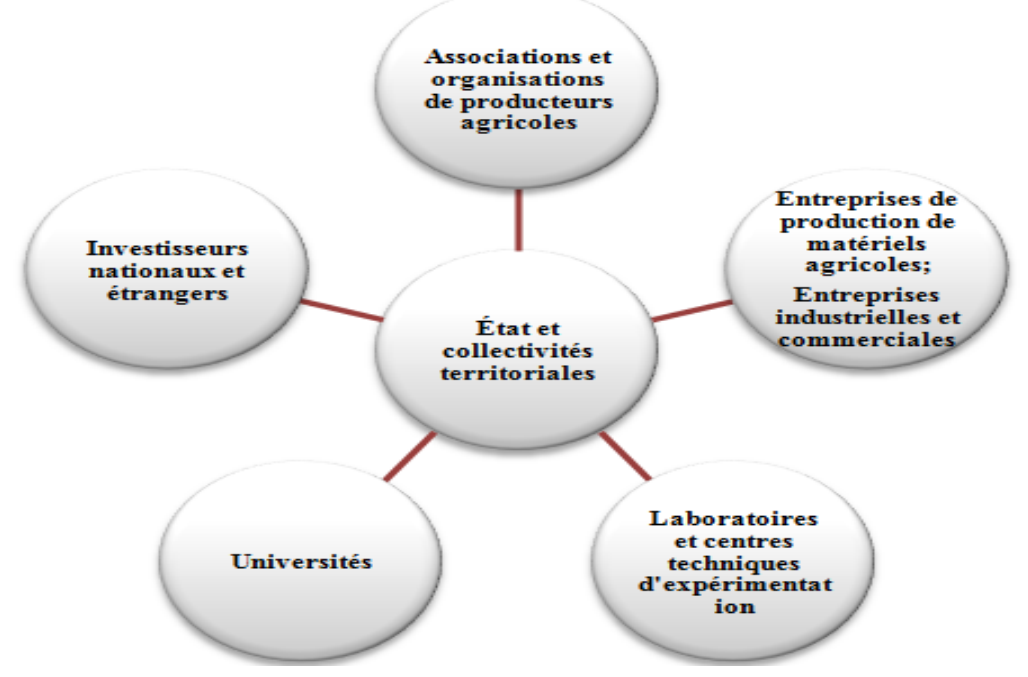

Figure 7. Schéma actuel de l'innovation dans le secteur agroalimentaire au Mali (Auteur à partir des politiques publiques de développement du secteur agricole)

\subsection{Analyse prospective des facteurs favorables au développement des capacités d'innovation dans le secteur agroalimentaire au Mali}

Cette étude permet de déceler les bases sur lesquelles une analyse prospective peut être réalisée en vue de créer les conditions favorables à l'acquisition, l'apprentissage, la transformation et la diffusion de nouvelles connaissances entre les différents acteurs. Les forces et les opportunités existent et peuvent servir de levier pour développer les capacités d'innovation dans le secteur agroalimentaire. Par contre, il y a des faiblesses pour lesquelles des solutions doivent être trouvées pour faire face aux menaces qui pèsent sur la mise en place de politiques publiques pérennes en matière de développement des capacités d'innovation.

\subsubsection{Forces et opportunités de développement des capacités d'innovation}

\subsubsection{Les forces}

- La disponibilité des acteurs (entreprises agricoles, institutions de financement, entreprises de fabrication ou d'assemblage de matériels agricoles) intéressés à investir pour le développement du secteur agricole, les ressources humaines (65\% ont moins de 25 ans en 2017, selon le PNUD). De plus en plus de jeunes s'orientent vers l'entrepreneuriat agricole en modernisant les activités d'élevage bovin, caprin, ovin, volaille, pisciculture, aquaculture. À cela s'ajoutent les potentialités agricoles (seulement $11,9 \%$ des terres sont mises en valeur), la disponibilité en eau de surface (fleuves Niger et Sénégal, nombreux lacs) et en eau souterraine.

- L'existence et l'augmentation du nombre de laboratoires, d'écoles professionnelles agricoles, de centres de formation en entrepreneuriat agricole et des centres techniques d'expérimentation peut 
contribuer à former et à mettre les compétences à la disposition du secteur agroalimentaire. De plus, le Comité National de la Recherche Agricole (CNRA peut s'appuyer sur l'Institut d'Economie Rurale (IER), le Laboratoire Central Vétérinaire (LCV), les écoles et facultés dans plusieurs domaines (médecine animale, vulgarisation agricole, recherche variétale, mise au point de techniques culturales, conception des ratios alimentaires pour les types d'élevage et d'animaux, amélioration des connaissances sur les systèmes de production, les espèces animales et les produits d'origine animale, les filières agricoles, etc.). Le CNRA peut ainsi s'appuyer sur les programmes de recherche afin de coordonner l'ensemble des activités d'innovation. La consolidation des démarches participatives (acteurs et utilisateurs des résultats de la recherche) peut contribuer à engager un processus d'apprentissage collectif, de réduire le fossé entre le monde académique et le monde économique et de faciliter la création de nouvelles connaissances et de savoirs.

\subsubsection{Les opportunités}

- La mise en œuvre de toute politique publique dépend de la volonté politique. Cette dernière existe étant donné les mesures prises les autorités pour mettre en place des programmes (PDA, LOA, CSCRP, PNISA) et des structures (agences de conseils et de promotion de l'entrepreneuriat, FCRIT...) afin d'acquérir les fonds, les technologies et les compétences pour développer le secteur.

- Les connaissances et les compétences acquises avec les routines organisationnelles doivent être valorisées. Il s'agit des actions tendant à mettre en place de nouveaux produits, procédés de fabrication, méthodes de commercialisation, formes d'organisation du travail, ou de développement de marchés, de partenariats (innovations frugales). Par exemple, les nouvelles pratiques (technique du goutte à goutte) visant à rationaliser la gestion des ressources en eau, la rotation des cultures, le recours à l'irrigation et les cultures de contre-saison, le développement de l'agriculture intensive, la combinaison des activités agricoles et d'élevage sur les mêmes surfaces en vue d'optimiser l'utilisation des ressources et de maîtriser la chaîne de valeur, réaliser des économies d'échelle, baisser les coûts de production et augmenter les revenus de producteurs. On assiste également à la création de petites unités de conservation et de transformation des produits agricoles.

- Au niveau de la transmission des connaissances et la gestion des entreprises agricoles, on peut détecter des innovations dites «sexuées ». Actuellement, aucune étude n'a été réalisée sur les apports des femmes dans l'innovation dans ce secteur au Mali. Toutefois, il serait opportun d'identifier leur rôle dans le processus d'apprentissage. On enregistre de plus en plus de femmes qui se lancent dans l'entrepreneuriat agricole et réussissent mieux en termes de gestion des coopératives ou d'unités agricoles. Elles représentent la majeure partie des clients des caisses de microfinance et remboursent mieux leurs dettes. Elles détiennent quasiment les petites unités de transformation des produits agricoles en produits alimentaires (confiture, jus de fruit, pâte alimentaire, boisson, conserve). Toutes ses activités ont des avantages multiples. Elles développent les circuits courts, rallongent le cycle de vie des produits agricoles, créent de nouvelles activités, de nouvelles techniques de production, de nouveaux emplois et des revenus, réduisent des inégalités sociales, améliorent les conditions de vie des populations. En 2015, ces activités sont considérées comme féminines en raison de leur part s'élevant à 50\% dans le secteur de l'alimentation (dont 49\% dans l'agriculture, 62\% dans la transformation alimentaire, $66 \%$ dans la commercialisation, $51 \%$ dans la restauration à domicile) ${ }^{12}$. L'optimisme vient du fait que la confiance, l'autonomie et les expériences acquises leur permettent de créer des conditions pour la construction des capacités d'innovation dans ce secteur. Elles sont en train de réorganiser les activités du secteur notamment les techniques d'apprentissage, l'optimisation $\mathrm{du}$ processus, et la contribution à la structuration du secteur. Mais pour le moment ces innovations paraissent diffuses.

\footnotetext{
12 Sources : À partir de ANSD (2015), Enquête nationale sur l'emploi 2015 (base de données) ; Banque mondiale (2017a), Enquête sur la mesure des niveaux de vie (base de données). Ces informations sont issues de la note rédigée par ALLEN, T., HEINRIGS, P., HEO, I., (2018), Agriculture, alimentation et emploi en Afrique de l’Ouest, Notes Ouest-Africaines N¹4, Édition OCDE, Paris. 
- La diaspora malienne peut être mobilisée pour combler le déficit de compétences dans tous les domaines. À ce titre, dans le cadre de l'aide française au développement au Mali, le Service de Coopération et d'Action Culturelle (SCAC) de l'Ambassade de France au Mali, a lancé dans les années 2000, le programme pluriannuel «300 jeunes cadres pour le Mali » destiné aux bacheliers maliens. Dès son lancement en 2018, 222 étudiants maliens en ont bénéficié, parmi lesquels 132 ont terminé leurs études, mais 44 seulement sont rentrés au Mali ${ }^{13}$. De plus, en 2010 , le SCAC a mis en place des bourses de spécialisation en santé destinées, d'une part, aux médecins maliens souhaitant faire des études de spécialisation dans une université Ouest-Africaine pour une durée de 3 à 5 ans et, d'autre part, à ceux en cours de spécialisation au Mali pour une formation médicale d'un an dans un centre hospitalier universitaire français en tant qu'interne. En 2014, 20 étudiants en médecine maliens ont bénéficié de ce programme. En 2015, a vu le jour le programme d'appui à l'enseignement supérieur à travers le financement de bourses de thèse en alternance destinées aux assistants de l'enseignement supérieur titulaires d'un master. Toujours dans le cadre de la mobilité internationale des étudiants et des jeunes chercheurs maliens, Campus France joue le rôle d'interface entre ces derniers et l'enseignement supérieur Français par la diffusion d'informations précises et actualisées sur les conditions de vie et d'accueil des étudiants étrangers en France. Depuis 2012, en moyenne $450^{14}$ jeunes vont étudier en France chaque année.

- La mise à profit des différents types de proximité (géographique, organisationnelle, cognitive, sociale, et institutionnelle) entre des acteurs de R\&D et les zones de production agricole. L'objectif est d'aboutir à la mise en place de systèmes de production localisés se basant sur une grande diversité d'acteurs locaux en particulier les centres de R\&D, les consommateurs, les entreprises, les pouvoirs public et institutions locales telles que chambres de commerce, ou coopératives de producteurs, d'une part; d'autre part, il s'agit de faciliter l'émergence d'effets externes positifs et d'effets d'agglomération [MAR 61] dont les acteurs pourraient bénéficier. Ce qui permettra de créer des écosystèmes innovateurs locaux grâce à un processus collectif d'apprentissage et d'actions impliquant un ensemble de relations formelles et informelles s'exprimant au sein de réseaux d'innovation localisés qui ne disposent pas à titre individuel des ressources suffisantes pour innover. Cette stratégie de coopération permet à la fois l'amélioration de la créativité et la réduction des risques et des coûts inhérents au processus d'innovation [MAI 95]. Ce type de milieux innovateurs dans le secteur agroalimentaire au Mali peut être obtenu grâce au groupement géographiquement proche d'entreprises et d'institutions associées, interconnectées au sein d'un champ particulier et liées par des éléments communs et par des complémentarités [POR 98] [POR 03]. L'idée est que la mise en synergie des entreprises locales est bénéfique dans la mesure où elle permet la diffusion des connaissances nécessaires au bon fonctionnement du système organisé.

\subsubsection{Obstacles et menaces dans la mise en œuvre d'une politique d'innovation au Mali}

\subsubsection{Les obstacles}

- Ils peuvent se résumer comme suit: l'insuffisance d'infrastructures (universités, centres de recherche, entreprises agricoles, routes, moyens de transport, communication, terres aménagées, etc.), entrave l'accès des produits agricoles aux différents marchés, entraîne l'augmentation des coûts de production et de transaction et le gaspillage alimentaire. Le manque d'industries de transformation des produits agricoles fait que les produits agricoles sont commercialisés, avec une faible valeur ajoutée constituant un manque à gagner pour les producteurs. Les faibles rendements agricoles résultent de la conjugaison de la dépendance aux aléas climatiques avec une pluviométrie en baisse de $30 \%$ sur les 30 dernières années [MIN 11], les sécheresses, les inondations, les invasions acridiennes régulières, la non maîtrise de l'irrigation ainsi que les difficultés d'accès aux intrants (terres, engrais).

\footnotetext{
${ }^{13}$ Source : https://ml.ambafrance.org/Les-missions-du-Service-de-cooperation-et-d-action-culturelle

${ }^{14}$ Ces informations ont été tirées sur le site de l'ambassade de France au Mali via le lien suivant : https://ml.ambafrance.org/Lesmissions-du-Service-de-cooperation-et-d-action-culturelle 
- L'isolement et le manque d'interactions entre les acteurs de la R\&D et du monde économique restreignent le financement et la valorisation de résultats de la recherche.

- La faible performance des entreprises agricoles se justifie par le faible niveau d'alphabétisation, de qualification, du système d'information et de statistiques agricoles, de mécanisation... Elle se répercute sur la capacité de gestion des organisations de producteurs et sur la valorisation des produits agricoles. À cela s'ajoute la non maîtrise des problèmes environnementaux (dégradation du couvert végétal et des sols, perte de la biodiversité, changement climatique, pollution, ensablement des cours les fleuves Niger et Sénégal, dégradation du cadre de vie, maladies animales), la volatilité des prix, l'instabilité des revenus agricoles, la faiblesse des échanges commerciaux avec les pays voisins... Une législation faible en matière de protection des droits de propriété intellectuelle et la gestion du foncier agricole freinent les investissements et l'innovation dans ce domaine. Toutes ces faiblesses sont dues à l'absence de politiques d'innovation dans le secteur.

\subsubsection{Les menaces}

Les menaces qui planent sur la mise en œuvre de ses politiques sont entre autres :

- L'insécurité qui sévit dans le pays depuis 2012 et les effets néfastes du changement climatique sont de réelles menaces qui empêchent la réalisation de toute activité viable et toute programmation des investissements en R\&D.

- Avec la mondialisation de l'économie, la concurrence étrangère peut être une menace étant donné la mobilité des compétences. En effet, il s'agit de chercher à résoudre de deux équations en termes de développement de la recherche domestique coûteuse et l'importation de produits manufacturés (alimentaires) et de technologies étrangères. De plus, le choix de l'importation des technologies pose le problème de la capacité d'absorption des entreprises locales, de la disponibilité des compétences susceptibles de les utiliser et les adapter aux réalités locales. Et aussi la menace des produits de contrefaçon est réelle, vu la faible protection des inventions locales et le choix des consommateurs pour les produits moins chers, peu importe la provenance.

- La disposition actuelle des zones de production agricoles fait qu'elles sont non seulement éloignées des centres de R\&D mais aussi il n'existe pas de relation formelle ou informelle [OYE 97] avec ces derniers leur permettant de bénéficier des externalités positives que chacun pourrait produire. Enfin l'importance de l'économie informelle est également une donnée à prendre en compte.

\section{Conclusion}

À la lumière de notre analyse, les problèmes entravant le développement des capacités d'innovation dans le secteur agroalimentaire malien peuvent se situer au niveau de la disponibilité $\mathrm{du}$ financement des activités de production, d'innovation et des compétences. À cela s'ajoute l'insuffisance de collaboration entre les acteurs de la même filière (conception, production, commercialisation des produits agricoles, activités annexes et connexes). Ce dysfonctionnement est lié d'une part au manque de concertation ou à la faible qualité des relations sociales, cognitives, commerciales ou financières entre ces acteurs (entreprises, centres techniques et laboratoires, universités, État, collectivités). D'autre part, il s'explique par les faibles flux informationnels, l'inefficacité des ressources et des potentialités malgré toutes les réformes et les mesures. Le paradoxe est que la force des relations sociales n'est pas mobilisée en faveur de la création de richesses. Cela peut s'expliquer entre autres par l'ignorance de l'importance des relations sociales dans l'activité économique, la prédominance du secteur informel faisant qu'elles sont moins mises en valeur et la méfiance dominant le climat des affaires. En effet, c'est l'État seul qui joue le rôle d'interface entre les acteurs et se retrouve être leur seul dénominateur commun. Cependant la prédominance de l'économie informelle pèse énormément sur la structuration du secteur. Alors pour 
que le secteur soit un véritable moteur de la croissance beaucoup de mesures peuvent être envisagées :

- L'évaluation des programmes précédents dans le secteur agricole est nécessaire pour identifier les raisons de leurs échecs, les insuffisances ainsi que les points positifs à prendre en compte dans l'élaboration des nouveaux programmes.

- La création, la réhabilitation, l'équipement en matériel de production, de conditionnement et de stockage de semences (maïs, mil, riz, oignon, haricot, orange, mangue, coton etc.), le financement et la dotation en ressources humaines qualifiées des centres régionaux de recherche agronomique dans les zones de production. Pour cela, les compétences de la diaspora malienne (près de 4000 étudiants ou diplômés ont été recensés en France, en 2019) peuvent être mobilisées pour résorber à court terme le déficit de compétences au niveau national. Cela permettra de tenir compte des contraintes locales et de faciliter la naissance de nouvelles zones de production de connaissances et de compétences.

- La valorisation des réalisations scientifiques à travers l'expertise des chercheurs, le dépôt de brevets, l'acquisition de licences, les démonstrateurs, les prototypes en vue d'applications industrielles, la mise à disposition des équipements coûteux et/ou rares non utilisés à plein temps des universités et laboratoires, les publications académiques ou grand public, la création de spin off ou de joint-ventures, les contrats de recherche, les conventions de partenariat, les projets de recherche collaborative, la consultance, les prestations de conseil ou de service, les conférences, les discussions informelles, la mobilité des chercheurs et/ou des étudiants alternants ou stagiaires... tout ceci pourrait contribuer à créer les conditions nécessaires à la construction des capacités d'innovation du pays.

- L'implantation d'acteurs pivots et de plateformes collaboratives dans les zones de production agricole peut faciliter la structuration, la coordination, la mutualisation des activités de production, d'innovation et la formation d'un véritable système. Le renforcement des capacités d'innovation de la Compagnie Malienne de Développement des Textiles (CMDT) dans les zones de production cotonnières et de l'office de Niger dans les zones de productions rizicoles est nécessaire.

- La modernisation du secteur agricole doit être renforcée par des mesures incitatives (exonérations fiscales, subvention, structures de conseils et d'accompagnement), par la garantie de l'accès à la terre, l'octroi de crédits à taux préférentiels aux entreprises souhaitant investir dans le secteur. Elle nécessite aussi le développement des marchés pour assurer des débouchés aux produits agroalimentaires locaux par la dotation en infrastructures (réseaux de communication, transport). De son côté, la diversification agricole (mil, maïs, fruits, arachide, beurre de karité, canne à sucre, sésame, blé etc.) doit être au cour de la politique publique pour répondre aux besoins des populations.

- La promotion d'une croissance durable, favorable aux plus démunis et créatrice d'emplois et d'activités génératrices de revenus pour les agriculteurs et le renforcement des bases à long terme du développement et de l'accès équitable aux services sociaux de qualité, supposent la modernisation des institutions de régulation et des processus de gouvernance des écosystèmes d'innovation. Pour la création d'un véritable système sectoriel d'innovation au Mali, en effet, les réflexions peuvent s'orienter d'une part vers la mise en valeur des notions «d'innovations frugales et informelles » et des « innovations sexuées » et, d'autre part, vers la mise en relation des centres de formation et de recherche avec les entreprises et les exploitants agricoles et avec les collectivités territoriales pour faciliter la production, la diffusion et la valorisation de connaissances à travers un processus interactif.

- Les acteurs (entrepreneurs, chercheurs, ingénieurs, etc.) peuvent saisir les nombreuses opportunités et les adapter aux réalités socioéconomiques dans le secteur en question. La mise en œuvre des mesures de politique publique nécessaires à la construction des capacités d'innovation dans l'agroalimentaire, greffées à celles qui existent en matière de développement en général, constituent une piste à explorer. Ces capacités peuvent contribuer à répondre à court et moyen terme 
aux besoins alimentaires de la population. Le renforcement des interactions entre les différents acteurs contribue au développement de leurs capacités d'innovation. Il a pour effets positifs la diversification, l'augmentation qualitative et quantitative de l'offre de produits agroalimentaires permettant de satisfaire la demande locale. Mais, le pessimisme provient de l'instabilité de l'environnement socioéconomique et sécuritaire depuis 2012 (conflits intermittents). Le risque des affaires devient ainsi incalculable. Un cercle vicieux supplémentaire se produit avec les conflits politico-militaires qui sont attisés par l'absence, l'inefficacité et l'insuffisance d'institutions et d'infrastructures socioéconomiques basiques dans de nombreuses localités du pays (schéma 8).

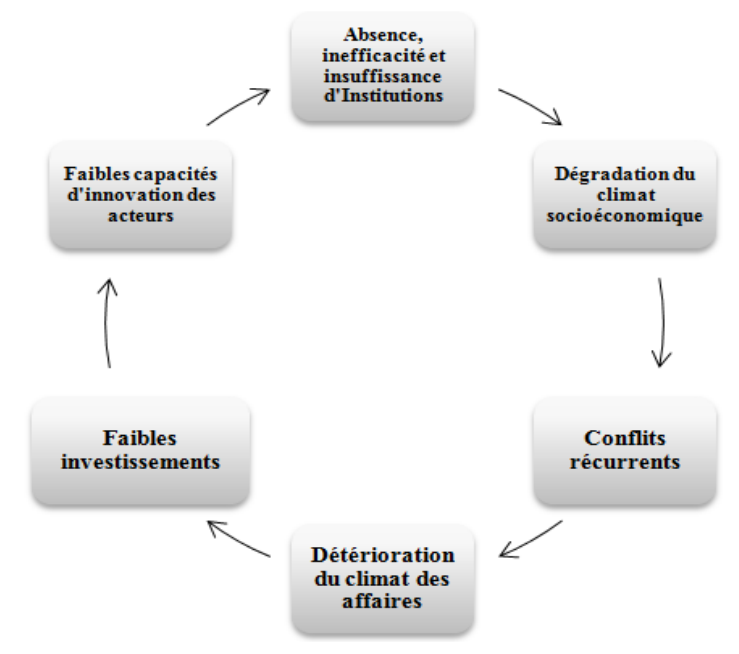

Figure 8. Le cercle vicieux de l'instabilité politico-économique

\section{Références}

[ALl 18] ALlEN, T., HEINRIGS, P., HEO, I., Agriculture, alimentation et emploi en Afrique de l'Ouest, Notes Ouest-Africaines $\mathrm{N}^{\circ} 14$, Édition OCDE, Paris, 2018.

[AMS 01] AMSDEN, A.H., The Rise of the "Rest", Challengers to the West from Late industrializing Economies, Oxford University Press, Oxford, 2001.

[ARO 01] AROCENA, R., SUTZ, J., Revisiting Nelson and Winter from the South: "Learning by Solving" in Underdeveloped Countries, DRUID conférence 2001, Danemark, Aalborg School, 2001.

[CARO 03] AROCENA, R., SUTZ, J., Subdesarollo e innovacion, navegando contra el viento, Cambridge, Cambridge University Press, 2003.

[BAN 18] BANQUE MONDIALE : http://www.banquemondiale.org/fr/topic/agriculture/brief/the-west-africaagricultural-productivity-program [Accédé le 08 Mai 2019], 2018.

[BCE 18a] BCEAO BANQUE CENTRALE DES ETATS DE L'AFRIQUE DE L'OUEST, Bulletin Trimestriel des Statistiques, Deuxième Trimestre, Abidjan, Côte d'Ivoire, 2018.

[BCE 18b] BCEAO BANQUE CENTRALE DES ETATS DE L'AFRIQUE DE L'OUEST., Bulletin Trimestriel des Statistiques, Quatrième Trimestre, Abidjan, Côte d'Ivoire, 2018.

[BCE 17] BCEAO BANQUE CENTRALE DES ETATS DE L'AFRIQUE DE L'OUEST., Rapport annuel 2017, Abidjan, Côte d'Ivoire, 2017.

[BEL 14] BELIERES, J F., Agriculture familiale et politique publique au Mali, Document de travail ART-Dev 20142013, version 1, Avril CIRAD, 2014.

[BEL 97] BELL, M., PAVITT ; K., Technological accumulation and industrial growth : contrast between developed and developing countries, dans Archibugi D., Michie, J.,(dir), Technology, Globalization and Economic Performance, P.83-138, Cambridge University Press, Cambridge, 1997.

[CAS 11] CASADELLA, V., Les SNI dans les PED, Editions Universitaires Européennes, Allemagne, 2011.

[CAS 18] CASADELLA, V., UZUNIDIS, D., Les capacités d'innovation comme préalable à la formation d'un système national d'innovation, ISTE OpenScience, Published by ISTE Ltd, London, UK, N 53, P 13-39, 2018. 
[CAS 17] CASADELLA, V., UZUNIDIS, D., National innovation systems of the south, innovation and economic development policies: A multidimensional approach, Journal of Innovation Economics \& Management, $\mathrm{N}^{\circ} 23$, pages 137 à 157, 2017.

[CAS 15] CASADELla, V., ZETING, L., UZUNIDIS, D., Développement économique et capacités d'innovation dans la mondialisation, Vol 1, Collection Innovation, Entrepreneuriat et Gestion, Série Smart Innovation, ISTE Editions, Great Britain, 2015.

[CAS 14] CASSIOLATO J.E, MATOS M.P, LASTRES H.M., Innovation Systems and Development, CURRIE ALDER B., KANBUR R., MALONE D.M, MDHORA R., International Development Ideas, Experience, and Prospects, Oxford: Oxford University Press, 2014.

[CIR 11] CIRAD et IER., Un observatoire des exploitations agricoles du Mali, Etude de conception dans le cadre du Projet PAPAM, 2011.

[CHA 12] CHAMINADE, A., INTARAKUMNERDB, P., SAPPRASERTC, K., Measuring Systemic Problems in National Innovation Systems: An Application to Thailand, Research Policy, 41, 1476-1488, 2012.

[CHA 09] CHAMINADE, C., LUNDVALL, B., JOSEPH, K., VANG, J., Innovation Policies for Development : Towards a Systemic Experimentation Based Approach, in Chaminade, C., Lundvall, B., Joseph, K., Vang, J., Handbook of Innovation Systems and Developing Countries, Cheltenham, Edward Elgar, 360-379, 2009.

[COH 89] COHEN W., LEVINTHAL D., Innovation and learning: the two faces of $R \& D$, Economic Journal, 99, P.569-596, 1989.

[DUF 06] DUFUMIER, M., BAINVILLE, S., Le développement agricole du Sud-Mali face au désengagement de l'État, Afrique contemporaine (n 217), p. 121-133, 2006.

[FAM 18] FAMINE EARLY WARNING NETWORK MALI (2018): http://fews.net/fr/west-africa/mali [Consulté le 23 Avril 2019], 2018.

[FER 18] FERRE, T., MEDAH. I., CRUZ J-F, DABAT. M-H., LE GAL. P-Y., CHTIOUI. M., DEVAUXSPATARAKIS. A., Innover dans le secteur de la transformation agroalimentaire en Afrique de l'Ouest, Published by EDP Sciences, 2018.

[FOO 18] FOOD AND AGRICULTURE ORGANIZATION, http://www.fao.org/ag/agn/nutrition/mli_fr.stm [Accédé le 04 Mai 2019], 2018.

[FOR 94] FORAY D. (1994), Les nouveaux paradigmes de l'apprentissage technologique, Revue d'Économie Industrielle, (69), p. 93-104, 1994.

[GRE 01] GREGERSEN, B., JOHNSON, B., Learning economy, innovation, systems and development, Paper prepared for the ESST Converge project, Danemark, 2001.

[HOB 95] HOBDAY, M., (1995), Innovation in East Asia: the challenger to Japan, Edward Elgar, Cheltenham, 1995.

[INN 18] INNOVATION ENVIRONNEMENT DEVELOPPEMENT AFRIQUE, http://www.iedafrique.org/Transfert-de-technologies-pour-le.html [Accédé le 07 Mai 2019], 2018.

[INS 18] INSTITUT NATIONAL DE LA STATISTIQUE DU MALI : http://www.instatmali.org/index.php/component/content/article/11-accueil/wwwjsc-53.html[Accédé le 04 Mai 2019], 2018.

[IPR 18] IPR/IFRA :https://docplayer.fr/4002161-Programme-de-formation-en-agroeconomie-a-l-ipr-ifra-dekatibougou.html[Accédé le 30 Avril 2019], 2018.

[JOUR 18] JOURNAL SCIENTIFIQUE ET TECHNIQUE DU MALI : http://www.jstm.org/financement-de-larecherche-scientifique-au-mali-46-projets-retenus-par-le-cnrst/AAccédé le 07 Mai 2019], 2018.

[JUM 01] JUMA, C.F., HONCA, D., HUETE-PEREZ, J. et al., Global Governance of technology : meeting the needs of developing countries, International Journal of Technology Management, vol.22, nº7-8, 2001.

[KIM 97] KIM, L., Imitation to innovation. The dynamics of Korea's Technological learning, Harvard University Press, Boston, 1997.

[LAL 02] LALL S, PIETROBELLI C., Falling to compete, Technology development and technology systems in Africa, Elgar, Cheltenham, 2002.

[LEB 93] LE BAS C., La firme et la nature de l'apprentissage, Economie et Sociétés, Série Dynamique Technologique et Organisation, (1), n 5, p. 7-24, 1993. 
[LEC 90] LE CHAU, DIOP. A., ET COULIBALY. I., Économie nationale et espace au Mali, Bamako, ORSTOM, p. 32-42, 1990.

[MAF 13] MAFAP, Revue des politiques agricoles et alimentaires au Mali, Série rapport pays SPAAA, FAO, Rome, Italie, 2013.

[MAH 87] MAHARAUX, A., Les industries du Mali, Cahiers d'outre-mer Nº 159, p. 237-264, 1987.

[MAI 95] MAILLAT, D., Milieux innovateurs et dynamiques territoriales, in A. RALLET, A, 1995.

[MAL 16] MALI FOOD AND SECURITY CLUSTER : https://fscluster.org/mali/document/cluster-sa-situation-dela-securite-2[Accédé le 28 Avril 2019], 2016.

[MAL 18] MALIPAGES :https://www.malipages.com/appel-offre/2016-10-service-de-formation-en-transformationagroalimentaire/[Accédé le 10 Mai 2019], 2018.

[MAR 61] MARSHALL, A., Principles of Economics, Macmillan, Londres, 8ème edition, 1890/1961.

[MIN 11] MINISTERE DE L'ENVIRONNEMENT ET DE L'ASSAINISSEMENTE, Gestion intégrée de la terre et de l'eau pour l'adaptation à la variabilité et au changement climatique: système d'information, Document de travail, Ministère de la sécurité et la protection civile, Sahara and Sahel Observatory, World Bank, Mali, 2011.

[MUC 03] MUChIE, M., GAMMERlTOFT, P., LUNDVAll, B., Putting Africa First: The Making of African Innovation Systems, Aalborg University Press, 2003.

[NAY 02] NARAYAN, N., PETESCH, P., Voices of the Poor: from Many Lands, Oxford University Press, Oxford, 2002.

[OCD 05] OCDE/EUROSTAT, Manuel d'Oslo : Principes directeurs pour le recueil et l'interprétation des données sur l'innovation, 3e édition, La mesure des activités scientifiques et technologiques, Éditions OCDE, Paris, 2005.

[OBG 95] OGBU O.M, OYEYINKA-OYELARAN B., MLAWA H., Understanding deindustrialization and technological stagnation in Sub-Saharan Africa: A framework, In Technology Policy and Practice in Africa: IDRC. Canada, 1995.

[OYE 97] OYELARAN-OYEYINKA, B., Nnewi: An Emergent Industrial Cluster in Nigeria, Ibadan, Technopol, 1997.

[PLA 14] PLAN NATIONAL D'INVESTISSEMENT DANS LE SECTEUR AGRICOLE AU MALI (PNISA). :http://www.passip.org/passip_intranet/pdf-intranet/Politique/9-

36\%20PNISA_version_finale_sept2014.pdf [Accédé le 30 Avril 2019], 2014.

[POR 03] PORTER, M.E., The economic performance of regions, Regional Studies, 37 (6 et 7), p. 549 579, 2003.

[POR 98] PORTER, M.E., Clusters and competition: new agendas for companies, governments and institutions, in M.E. PORTER, On Competition, Harvard Business School Press, Boston, MA, 1998.

[SEN 00] SEN, A., Development as Freedom, Anchor Books, New York, 2000.

[TOU 14] TOUZARD, J-M., TEMPLE, L., FAURE, G., TRIOMPHE, B., Systèmes d'innovation et communautés de connaissances dans le secteur agricole et agroalimentaire, Innovations, $\mathrm{N}^{\circ} 43$, pages 13 à 38, 2014.

[WIL 81] WILLIAMSON, O., The Economics of Organization, the Transaction Cost Approach, The American Journal of Sociologyy, vol 87 n³, 1981.

[ZOG 17] ZOGNING, F., MBAYE, A-A., UM-NGOUEM, M-T., L'économie informelle, l'entrepreneuriat et l'emploi, Les Edition JFD, Québec, Canada, 2017. 